\title{
It Takes Two to Kiss, but Does it Take Three to Give a Kiss? Categorization Based on Thematic Roles
}

\section{Citation}

Wittenberg, Eva, and Jesse Snedeker. 2013. It takes two to kiss, but does it take three to give a kiss? Categorization based on thematic roles. Language and Cognitive Processes: 1-7.

\section{Published Version}

doi:10.1080/01690965.2013.831918

\section{Permanent link}

http://nrs.harvard.edu/urn-3:HUL.InstRepos:11315417

\section{Terms of Use}

This article was downloaded from Harvard University's DASH repository, and is made available under the terms and conditions applicable to Open Access Policy Articles, as set forth at http:// nrs.harvard.edu/urn-3:HUL.InstRepos:dash.current.terms-of-use\#OAP

\section{Share Your Story}

The Harvard community has made this article openly available.

Please share how this access benefits you. Submit a story.

Accessibility 
RUNNING HEAD: Categorization Based on Thematic Roles

It Takes Two To Kiss, But Does It Take Three To Give A Kiss?

Categorization Based On Thematic Roles

Eva Wittenberg ${ }^{1,2}$ and Jesse Snedeker ${ }^{1}$

${ }^{1}$ Harvard University; ${ }^{2}$ Universität Potsdam

Corresponding Author:

Eva Wittenberg

Department of Psychology

Harvard University

33 Kirkland St. 1068

Cambridge, MA 02138

Tel: 617-304-7925

eva@wjh.harvard.edu 
Language is characterized by broad and predictable mappings between meaning and syntactic form. Transitive sentences typically encode two-participant events while ditransitives typically encode three-participant events. Light-verb constructions, however, systematically violate these mappings; for example, some have ditransitive syntax ('Romeo is giving Juliet a kiss') but describe what appear to be agent-patient events (Romeo kissing Juliet). We used a conceptual sorting task to explore whether this non-canonical mapping influenced the interpretation of these sentences. Participants were trained to sort events by the number of thematic roles they encoded. After a training phase with only pictures, they sorted a mix of pictures and written sentences, including transitive agent-patient sentences, ditransitive sourcetheme-goal sentences, and ditransitive light-verb constructions. Events described by light-verb constructions were most often grouped with agent-patient events but were sometimes grouped with source-theme-goal events. A control condition using the transitive/intransitive alternation for joint action verbs (e.g., 'meet') demonstrates that this is not attributable to misconstruing the task as syntactic sorting. We conclude that non-canonical mappings between meaning and form can affect event construal, but syntactic form does not solely determine the construal that is chosen.

Keywords: Events, Argument Structure, Conceptualization, Sorting Task, Syntax, Semantics, Light-Verb Constructions 
Languages are characterized by robust mappings between the structure of a sentence and its meaning. One manifestation of this is the systematic mapping between the syntactic roles in a sentence and the semantic roles of the participants in the event. For example, transitive sentences such as 'Romeo is kissing Juliet' have a subject and an object argument, which denote the agent and the patient. Likewise, a ditransitive frame with a subject and two objects, such as in 'Romeo sent a letter to Juliet' encodes a source, a theme, and a goal. These canonical mappings allow us to quickly identify who did what to whom and thus are helpful both in acquisition and in processing. ${ }^{i}$ The uniformity in the mapping between syntactic arguments and thematic roles is so pervasive that many linguistic theories take it as an axiom (Baker 1988; Chomsky 1981/1993). For example, Construction Grammar (Bencini \& Goldberg, 2000; Johnson \& Goldberg, 2012) argues that just encountering a transitive syntactic frame steers listeners towards assigning agent and patient to subject and object.

But curiously, in addition to these broad and systematic regularities, languages also feature a class of narrower but very frequent constructions that violate these patterns: light-verb constructions. In light-verb constructions such as 'Romeo gave a kiss to Juliet', there is a subject, a direct object, and a prepositional object. These syntactic arguments correspond to 'Romeo', 'a kiss', and 'Juliet'. But what are their thematic roles? The syntactic frame would suggest that the event is an instance of 'giving' that has a source, theme, and goal. However, given that the same event can be described by the paraphrase 'Romeo kissed Juliet', one might conclude that 'giving a kiss' is simply a 'kissing' event that only has an agent and a patient. 
Which of these thematic structures is correct? We see three possibilities: 1) The different-syntax hypothesis: differences between the ditransitive light and nonlight constructions stem from the syntax: 'giving a kiss' is syntactically different from the standard ditransitive 'giving a book' in a way that makes 'kiss' part of the predicate and thus inaccessible to thematic role assignment (Hale \& Keyser, 1993, 2002). As a result, 'give a kiss' is syntactically transitive and subject to a canonical mapping from transitive sentences to agent-patient events. 2) The differentsemantics hypothesis: the syntax of 'giving a kiss' is the same as 'giving a book', and the standard thematic role assignments apply. As a result, 'Romeo is giving a kiss to Juliet' is actually a source-theme-goal event, instead of an agent-patient event like 'Romeo kisses Juliet.' 3) The different-mapping hypothesis: the syntax of both ditransitive constructions is the same, but the light-verb construction maps onto an agent-patient event, resulting in a non-canonical mapping.ii

A prediction of the different-syntax hypothesis has been explored using syntactic priming (Wittenberg \& Snedeker, 2011). It is well established (cf. Bock, 1986; Bock \& Loebell, 1990) that, in a variety of tasks, people will use more double-object sentences when primed by double-object sentences ('Romeo gives Juliet a rose') than when primed by prepositional-object sentences ('Romeo gives a rose to Juliet'). We tested whether light-verb ditransitives would prime non-light ditransitives as effectively as other non-light ditransitives would. If light-verb constructions have a different syntactic structure from non-light constructions, they should be less effective primes for non-light constructions, since the degree of representational overlap is decreased. However, there was robust priming both from light to non-light 
constructions and also vice versa, suggesting that light and non-light constructions have the same syntactic form.

The present study explores the different-semantics and different-mappings hypotheses. The different-semantics hypothesis states that the syntactic form of the ditransitive leads to the conceptualization of 'give a kiss' as a source-theme-goal event. The challenge for this hypothesis is to explain how kissing and giving a kiss can have different semantic interpretations when they often pick out the same spatiotemporal chunks of experience. One possibility is that a given piece of experience can be construed in different ways resulting in distinct event structures which are expressed with different syntactic frames. For example, the ditransitive construction may pick out a construal of kissing that involves transfer.

Similar changes in event construal have been found with other syntactic alternations. Using the locative alternation ('fill the glass with water/fill water into the glass'), Gropen, Pinker, Hollander and Goldberg (1991) found that subtle changes to the structure of an event affected which syntactic form was used to describe that event. They asked adults and children to describe scenes where an object moved onto a surface. Whenever the motion itself was made more salient, participants tended to encode the object as direct object; whenever the surface changed its state, participants tended to encode the surface as direct object. Thus, even though these constructions refer to many of the same events, changing subtle aspects of the event representations can affect which form of the locative people use. The differentsemantics hypothesis proposes that comparable light and non-light constructions also have different meanings. When using the ditransitive form of 'give a kiss', people 
construe kissing as a source-theme-goal event, with the kiss as a third entity that is transferred from Romeo to Juliet, while the transitive encoding in 'kiss' results in an agent-patient construal of the event.

The different-mapping hypothesis, in contrast, assumes that the light-verb construction and base verb express the same event representation. On this hypothesis, the mapping mechanism between syntax and semantics must be flexible enough to assign agent and patient onto Romeo and Juliet, just as the usual mapping of agent to subject and patient to object can be overridden in passive voice, or with experiencer-stimulus verbs such as 'fear' (Hartshorne \& Snedeker, 2012).

To explore these hypotheses, we used an event-categorization task. A similar task was used by Bencini and Goldberg (2000). In their study, participants were asked to sort sentences that varied in both the verb that was used and the construction in which it appeared. For example, the verb 'throw' appeared in a transitive ('Anita threw the hammer'), ditransitive ('Chris threw Linda the pencil'), caused motion ('Pat threw the keys onto the roof') and resultative construction ('Lyn threw the box apart'). They found that participants relied on both verb meaning and construction type to sort the stimuli, indicating that both factors influence event categorization. Crucially, in Bencini and Goldberg's (2000) study, the different constructions had meanings that were uncontroversially distinct (they conveyed different information and picked out different categories of events). In the case of light-verb vs. base-verb constructions, it is not clear whether the meanings of the two forms are truly different.

In the present study, we asked participants to sort non-linguistic events 
according to the number of thematic roles involved. After a purely non-linguistic training phase, we introduced sentences that had three roles ('Anne is giving Julius a book'), two roles ('Walter is eating an apple'), one role ('Charles is sleeping'), or were either light-verb constructions ('Romeo is giving Juliet a kiss') or their corresponding base verbs ('Romeo is kissing Juliet').

If light-verb constructions are given a meaning that is consistent with their syntax, then participants should interpret the event noun as a separate role in the event and categorize events described by light-verb constructions with three-role events (different-semantics hypothesis). However, if light-verb constructions have the same thematic roles as the base verb constructions, despite their syntactic form, then participants should not treat the event noun as a separate role in the event, and should categorize light-verb constructions with two-role events (different-mappings hypothesis).

These predictions assume that participants can be trained to sort on the basis of semantic structure rather than syntax. To ensure that this is true, we included two additional types of stimuli: Sentences that described joint actions either in a transitive ('Jan meets Elsa') or intransitive form ('Jan and Elsa meet'). These symmetrical actions involve two people, Jan and Elsa. While there is considerable dispute as to the nature of the thematic roles involved (Carlson, 1998; Gleitman, Gleitman, Miller \& Ostrin, 1996), these verbs necessarily require two participants (*Jan is meeting). Thus if semantic structure is driving categorization, we expected that they would both be grouped with two-participant events. If syntax alone is driving categorization, participants should sort these items in the two-role group 
when they occur in transitive syntax and into the one-role group when they are in intransitive syntax.

It is also important to rule out sorting based on the number of concrete entities involved in the event, rather than the number of thematic roles. For this reason, we included items where the number of roles and the number of concrete entities were inconsistent, with more than one entity playing a given role (for example, 'The traffic lights are flashing').

\section{METHODS}

Participants

Thirty-six English native speakers from the Harvard study pool (mean age: 19.8, 30\% male) participated for study credit. Four of these participants were excluded from the analysis, since their performance was lower than $66 \%$ on the filler items.

Materials

We constructed ten pairs of light-verb (1a) and base-verb sentences (1b) and ten pairs of transitive (1c) and intransitive joint actions (1d):

(1) a. The teenager is giving his rival a kick.

b. The teenager is kicking his rival.

c. The father is cuddling the baby.

d. The father and the baby are cuddling.

The light-verb constructions all used 'give' as main verb, but one of the objects constituted the main semantic predicate of the sentence (such as 'kiss' in 'give a 
kiss'). The base-verb sentences used the same agents as their light-verb counterparts, but instead of having a theme, the theme's base verb served as the main predicate for the sentence.

One factor that could influence event construal is frequency. To take this possibility into account, we conducted a Google search for the light- and base-verb pairs. Most of the material indexed in Google is not edited, providing global input statistics about everyday language use. Our search strings were the exact form of the respective construction, but with either either "me" or "him" as object ("is kissing me/him"; "is giving me/him a kiss"). This search revealed that among all relevant descriptions of one event ("kissing"), light-verb constructions were used between 1\% and $50 \%$ of the time (mean: $28 \%$, SD: $25 \%$ ).iii

We created ten examples of one-role, two-role, and three-role sentences to serve as a baseline for evaluating participants' performance. In half of the sentences, the number of thematic roles and the number of entities converged (consistent sentences), and in the other half, there were more entities than roles (inconsistent sentences). All three-role sentences had animate agents, animate recipients, and inanimate themes of varying degrees of concreteness. ${ }^{\text {iv }}$ All two-role sentences used change-of-state verbs that cannot enter light-verb constructions. We also commissioned 20 pictures showing three-role scenes, 20 pictures showing two-role scenes, and 20 pictures showing one-role scenes (see Figure 1 for examples). Three additional pictures were created for the introduction, and 12 for the training phase (one consistent and one inconsistent example each for the one-, two-, and three-role picture categories). 


\section{(Figure 1 about here)}

40 native English speakers, recruited via Amazon Mechanical Turk, rated the sentences for naturalness on a scale from 1 to 9 ( 9 was most natural). There were no differences in rating scores between sentence types $(\mathrm{M}=8.0, F(9,60)=1.60, p=.13)$.

To make sure that participants interpreted the pictures as we intended, 15 workers on Amazon Mechanical Turk wrote a sentence to describe each one. A trained research assistant coded the sentences as correct if they encoded both the intended action and the intended participants, but included no additional participants. The average score across pictures was $84 \%$, and the scores did not differ significantly between picture types, $(F(5,72)=.83, p=.53)$. Critically, there were no differences between consistent and inconsistent pictures (one-role pictures: $F(1,24)=.71, p=.79$; two-role pictures: $F(1,24)=.77, p=.39$; three-role pictures: $F(1,24)=.00, p=.99)$.

We created two lists that showed half of the critical pairs as base verbs and half of them as light-verb constructions and showed half of the joint action pairs in transitive form, and the other half in intransitive form. Lists were counterbalanced such that no participant saw both items in one pair. In total, each list consisted of 60 pictures and 50 sentences.

Procedure

The experiment was executed using ePrime. Figure 1 illustrates the stimuli for each phase. First, participants were introduced to the concept of thematic roles, and were told that they should base their sorting behavior on how many thematic roles were involved in the event, not how many entities were involved.v This short 
introduction was followed by a training phase consisting of twelve pictures, which could be sorted by clicking on one of three reference pictures on the bottom of the screen. Participants had to sort all training pictures correctly before advancing to the test phase.

In the test phase, the participants continued sorting the items, which now included both pictures and sentences, into the three categories by clicking on the appropriate example picture, but without any feedback from the experimenter. There were no pictures paired with the test sentences. Post-test questionnaires showed that no participant guessed the goal of the experiment.

\section{RESULTS.}

During the test phase, inconsistent pictures were sorted less accurately than consistent pictures $(77 \%$ vs $87 \%, F(1,68)=21.3, p<.001)$, but performance was well above chance for both (consistent pictures: $F(1,190)=1,197.2, p<.001$; inconsistent pictures: $F(1,190)=379.3, p<.001)$, showing that participants primarily responded based on the number of arguments rather than the number of entities.

Performance on the baseline sentences was also good: Participants correctly sorted $92 \%$ of the consistent sentences and $84 \%$ of the inconsistent sentences, again with a significant difference between the two categories $(F(1,68)=10.3, p<.001)$, but above chance performance in both cases (consistent sentences: $F(1,68)=1,458.7$, $p<.001$; inconsistent sentences: $F(1,190)=923.3, p<.001)$.

Figure 2 shows how critical items were sorted. The data were analyzed using separate multilevel logistic regression models to predict the probability of sorting 
items into a given pile (e.g., the two-role group), with random slopes for subjects and items, and sentence type as a fixed effect. For the light-verb constructions, we also included the relative frequency of the light-verb construction in the corpus as a predictor (light-verb construction/light-verb construction + base verb). We found that proportional frequency of the light-verb construction was not a reliable predictor of sorting behavior $(z s<.38, p s>.69)$ and thus we removed it from subsequent analyses.

(Figure 2 about here)

Both types of joint actions were consistently sorted into the two-role group (intransitive: $89 \%$, transitive: $92 \% ; z=-1.34, p>.18$ ). Thus, participants were able to ignore syntactic variation and sort on the basis of thematic roles.

Light-verb constructions were more likely to be classified as three-role events than base-verb sentences ( $23 \%$ vs. $5 \% ; z=3.35, p<.001)$. They were also less likely than base-verb sentences to be construed as two-role events (75\% vs. $94 \% ; z=-4.06$, $p<.001)$. Thus the use of the light-verb construction affects sorting behavior.

Crucially, light-verb constructions were not simply treated as ditransitives: they were far less likely to be treated as three-role events than the three-role consistent sentences $(z=-8.51, p<.001)$, which were placed in this group $78 \%$ of the time. There was also a difference between light-verb constructions and two-role inconsistent sentences $(z=2.88, p<.01)$, which were sorted as three-role events only $7 \%$ of the time, despite having three participants. Thus, the sorting of light-verb constructions with three role events was not simply the result of counting nouns.

In addition, we correlated the number of three-role sortings of light-verb 
constructions with the number of one-role sortings for Joint Action Intransitives across participants. If our results reflected the use of syntax as a sorting strategy (on a per participant basis), then these two measures should be related. However, the correlation coefficient was significant and small $(r=0.0016 ; t=0.009 ; p>.99)$. Thus, it is unlikely that the results were driven by particular participants sorting according to syntactic structure.

\section{DISCUSSION.}

In this study, we found that participants sorted light-verb constructions differently from both base-verb sentences and three-role events, suggesting a threeway distinction between canonical transitive agent-patient events, events denoted by light-verb constructions, and canonical ditransitive source-theme-goal events.

This particular pattern, by itself, could have been interpreted as evidence of a strategy to sort based on syntactic structure alone. This possibility, however, is ruled out by the joint action conditions: participants consistently sorted intransitive joint actions as two-role events, despite their syntactic similarity to other intransitives. Also, it is clear that participants did not sort based on the number of entities or nouns, as shown by the reliable difference between light-verb and three-role consistent sentences, as well as performance on the two-role inconsistent sentences.

These findings have two limitations. First, we explicitly trained participants to pay attention to semantic roles and provided extensive instructions (on the effect of instructions, see Bencini \& Goldberg, 2000), thus we cannot say how sorting would occur in the absence of training. Nevertheless, this method is commonly used to 
understand how learners conceptualize potentially ambiguous stimuli (see e.g., Hommel, Alonso \& Fuentes, 2003; Chambers, Onishi \& Fisher, 2010). When participants are trained using one type of stimuli (e.g., transparent one-, two- and three-argument pictures) and then tested with very different stimuli (e.g., light-verb constructions), we can learn how they generalize their training. Second, participants' performance when sorting on one semantic dimension (thematic roles) cannot tell us about other semantic differences between light and base verbs, such as aspect or telicity. While we suspect that other differences exist (see Butt, 2010), the current data speak only to differences in the number of semantic roles in the event representation.

Taken at face value, this data seems to provide some support for both the different-mappings and different-semantics hypotheses. The evidence for different mappings is clear: on $75 \%$ of the trials, participants interpreted light-verb sentences as agent-patient events despite their ditransitive syntax. The evidence for different semantics is more subtle: on a minority of the trials, participants classified light-verb sentences as having three arguments, despite the event structure of the base verb.

We see three ways to reconcile these effects: 1) Both hypotheses could be true. Light-verb constructions could typically involve non-canonical mappings to the event structure of the base verb, but there could also be some cases where they are interpreted with a canonical mapping via the light verb, resulting in a categorically different interpretation. 2) Encountering the verb 'give' causes a thematic gardenpath effect; that is, the reader assigns source, theme and goal roles before reconsidering the nature of the event, and this representation lingers on (cf. Ferreira, 
2003). 3) Light-verb constructions may intrinsically have two different, shared argument structures that are active at the same time: agent-patient (from the noun) and source-theme-goal (from the verb). This view, sometimes called 'argument sharing' (Jackendoff, 1974; Butt, 2010), is supported by studies that find that lightverb constructions incur processing costs even when the event nominal precedes the verb, and thus the verb cannot lead the reader down the garden path (cf. Wittenberg, Jackendoff, Paczynski, Kuperberg, Snedeker \& Wiese, in press). Specifically, processing light-verb constructions leads to longer reaction times in cross-modal lexical decision tasks and a sustained negativity in ERP, compared with non-light constructions.

Our findings contribute to a small literature on the relationship between the conceptualization of events and their syntactic encoding. In some cases, these effects reflect the information that is present or absent in the sentence. For example, when agents are expressed, they are seen as more causally responsible (Faussey \& Boroditsky, 2010). In other cases, the effects reflect differences in the event structures encoded by sentences that might otherwise seem to be synonymous (e.g., Gropen et al., 1991). The present study also examines apparently synonymous sentences and finds subtle differences in conceptualization: light-verb constructions are sometimes interpreted as having three participants. This difference suggests that canonical mappings play a role in interpretation, even for non-canonical constructions where the result of this mapping is implausible.

Converging evidence for the subtle influence of syntactic structure onto conceptualization comes from a study by Majid, Sanford \& Pickering (2007), which 
investigated the influence of verb frame on story continuations. These continuations were coded for whether participants mentioned causes or effects of the event described. Transitive base verbs elicited few effect continuations and ditransitive nonlight verbs elicited many. Curiously, the light-verb constructions fell between the others. Like the current study, this suggests a role for syntactic structure in how people conceptualize linguistically-encoded events.

Such effects are surprising given the frequency of light-verb constructions; adults have ample practice with these collocations, and yet they still appear to experience some influence from the canonical ditransitive mapping. 


\section{ACKNOWLEDGEMENTS:}

We thank Adele Goldberg, Manizeh Khan, Roger Levy, Hugh Rabagliati, Heike Wiese and two anonymous reviewers for helpful comments and suggestions, as well as Talia Fox and Zheng Zhang for assistance with data collection and John Campbell for creating the pictorial stimuli. This research was supported by NSF Grant 0921012 to J.S. and a doctoral scholarship by the German National Academic Foundation to E.W. 


\section{REFERENCES:}

Baker, Mark C. 1988. Incorporation: A Theory of Grammatical Function Changing. Chicago, Illinois: The University of Chicago Press.

Bencini, G. M., \& Goldberg, A. E. (2000). The contribution of argument structure constructions to sentence meaning. Journal of Memory and Language, 43(4), 640651.

Bock, J. K. (1986). Syntactic persistence in language production. Cognitive Psychology, 18(3), 355-387.

Bock, K., \& Loebell, H. (1990). Framing sentences. Cognition, 35(1), 1-39.

Butt, M. (2010). The Light Verb Jungle: Still Hacking Away. In M. Amberber, M. Harvey and B. Baker (eds.) Complex Predicates in Cross-Linguistic Perspective, 48-78. Cambridge University Press.

Carlson G. (1998). Thematic roles and the individuation of events. In S. Rothstein (ed). Events and Grammar. Kluwer. pp 35-51.

Chambers, K. E., Onishi, K. H., \& Fisher, C. (2010). A vowel is a vowel: Generalizing newly-learned phonotactic constraints to new contexts. Journal Of Experimental Psychology. Learning, Memory, And Cognition, 36(3), 821-828.

Chomsky, N. (1981/1993). Lectures on Government and Binding: The Pisa Lectures. Mouton de Gruyter.

Fausey, C. M., \& Boroditsky, L. (2010). Subtle linguistic cues influence perceived blame and financial liability. Psychonomic Bulletin \& Review, 17(5), 644-650.

Ferreira, F. (2003). The misinterpretation of noncanonical sentences. Cognitive Psychology, 47(2), 164-203. 
Gleitman, L. R., Gleitman, H., Miller, C., \& Ostrin, R. (1996). Similar, and similar concepts. Cognition, 58(3), 321-376.

Gropen, J., Pinker, S., Hollander, M., \& Goldberg, R. (1991). Affectedness and direct objects: The role of lexical semantics in the acquisition of verb argument structure. Cognition, 41(1), 153-195.

Hale, K. L., \& Keyser, S. J. (1993). On argument structure and the lexical expression of syntactic relations. The view from building 20: Essays in linguistics in honor of Sylvain Bromberger (53-100). Cambridge, MA: MIT Press.

Hale, K. L., \& Keyser, S. J. (2002). Prolegomenon to a theory of argument structure. Cambridge, MA: MIT Press.

Hartshorne, J. K., \& Snedeker, J. (2012). Verb argument structure predicts implicit causality: The advantages of finer-grained semantics. Language and Cognitive Processes, DOI:10.1080/01690965.2012.689305

Hommel, B., Alonso, D., \& Fuentes, L. (2003). Acquisition and generalization of action effects. Visual cognition, 10(8), 965-986.

Jackendoff, R. (1974). A deep structure projection rule. Linguistic Inquiry, 5(4), 481505.

Johnson, M.A. \& Goldberg, A. E. (2012): Evidence for automatic accessing of constructional meaning: Jabberwocky sentences prime associated verbs, Language and Cognitive Processes, 1-14.

Majid, A., Sanford, A. \& Pickering, M. (2007). The linguistic description of minimal social scenarios affects the context of causal inference making. Journal of Experimental Social Psychology, 43, 918-932 
Piñango, M. M., Mack, J., and Jackendoff, R. (in press.) "Semantic combinatorial processes in argument structure: Evidence from light verbs". Proceedings of the annual meeting of the Berkeley Linguistics Society, 2006.

Wittenberg, E., and Snedeker, J. (2011). Syntactic Priming Across Constructions: Light Verbs. Poster, AMLaP Conference, Paris.

Wittenberg, E., Jackendoff, R., Kuperberg, G., Paczynski, M., Snedeker, J \& Wiese, H. (in press). The Processing and Representation of Light Verb Constructions. In: Bachrach, A., Roy, I. and Stockall, L. (eds.). Structuring the Argument. John Benjamins. 


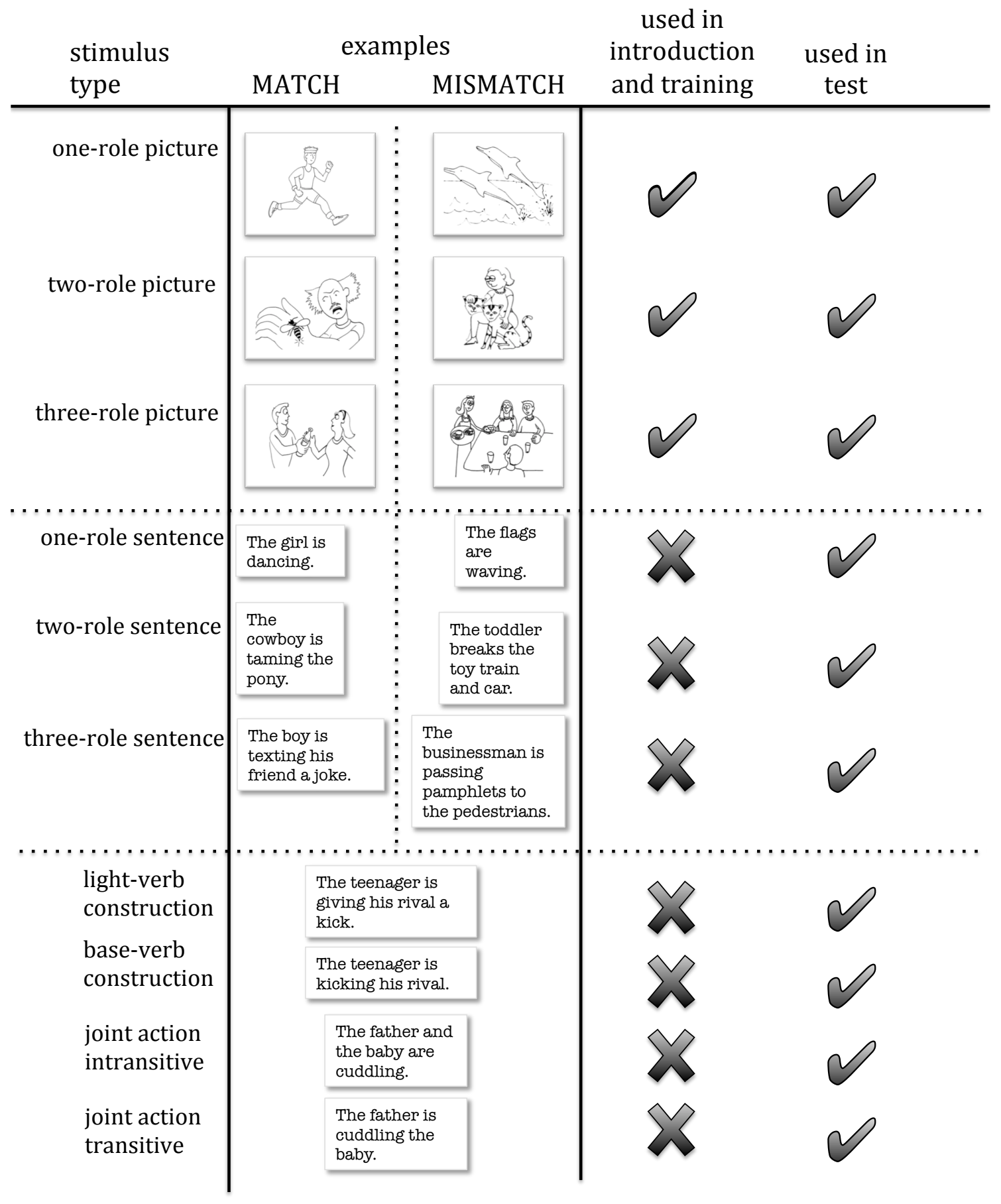

Figure 1: Procedure of the experiment: Participants were introduced to and trained on pictures only. In the experimental phase, they also saw sentences. 


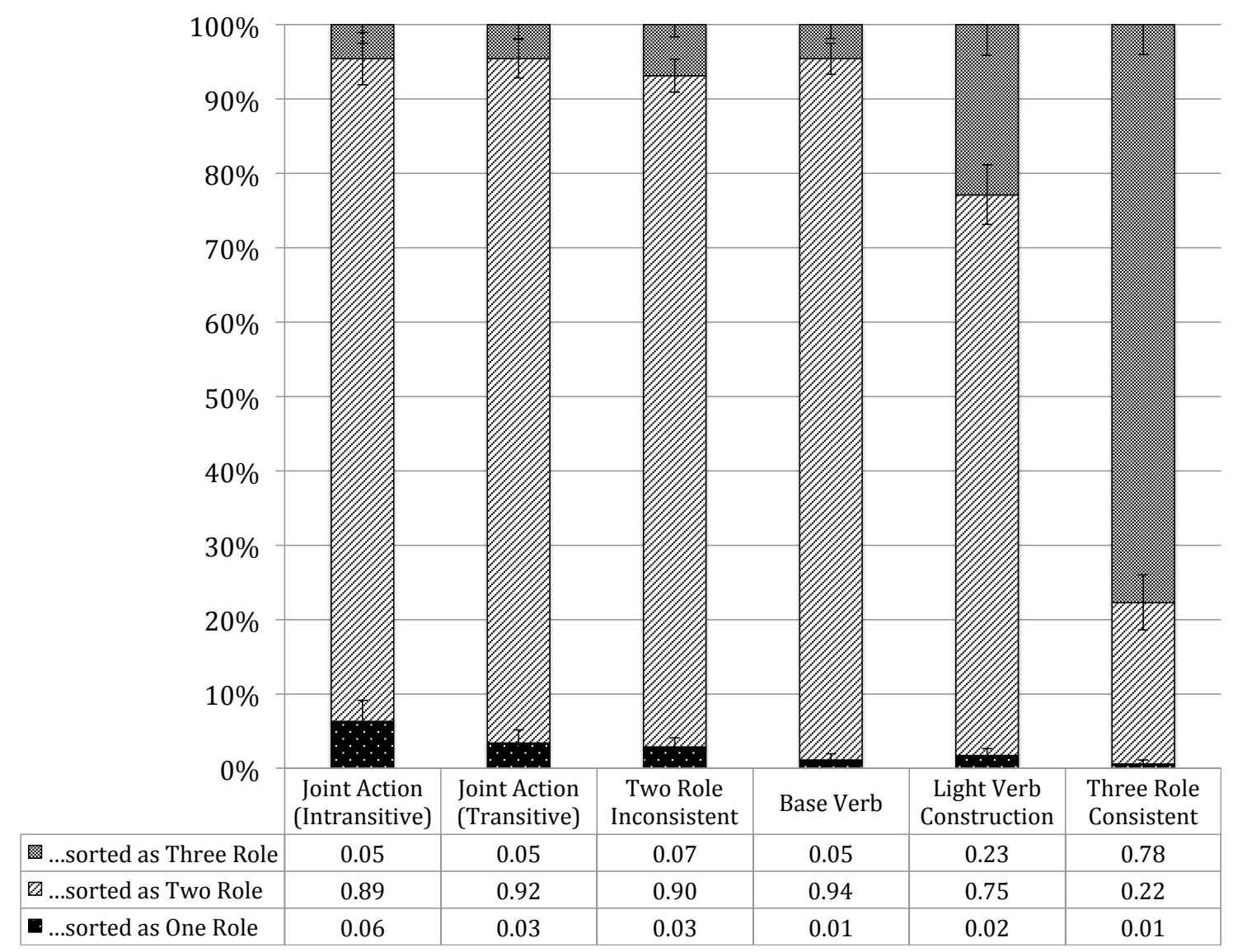

Figure 2: Categorization patterns for the critical sentences, with Standard Errors. The differences between light-verb constructions, and base verb, two role inconsistent, and three role consistent sentences were significant; the differences between the two types of joint actions were not. 


\section{FOOTNOTES:}

\footnotetext{
${ }^{\mathrm{i}}$ By canonical we simply mean the mappings that preserve a one-to-one correspondence between thematic roles and noun phrases. We do not intend to imply that these mappings are privileged in processing or acquisition.

${ }^{\text {ii }}$ For the sake of simplicity, we'll assume that a semantic structure gets translated directly into an event representation, while acknowledging that the latter is much richer than the former. Our discussion will be agnostic about the semantics-conceptual structure divide, since this does not bear on our question.

${ }^{\text {iii }}$ For per-item frequency data, see http://dvn.iq.harvard.edu/dvn/faces/study/StudyPage.xhtml?globalId=hdl:1902.1/19337

${ }^{\text {iv }}$ See for full stimuli list

http://dvn.iq.harvard.edu/dvn/faces/study/StudyPage.xhtml?globalld=hdl:1902.1/19337

v For specific instructions, please see

http://dvn.iq.harvard.edu/dvn/faces/study/StudyPage.xhtml?globalId=hdl:1902.1/19337
} 